\title{
Erratum to: Structure of High Latitude Currents in Magnetosphere-Ionosphere Models
}

\section{Wiltberger ${ }^{1}$}

Published online: 10 August 2016

(C) Springer Science+Business Media Dordrecht 2016

Due to an unfortunate turn of events the Acknowledgements section was published without mention of the "US Geological Survey Geomagnetism Program". Please find the correct version of the Acknowledgements in this erratum that should be regarded as the final version.

Acknowledgements Support for this paper was provided by NASA grant NNX14AI13G and NSF grants M0856145, AGS-1242011, and the US Geological Survey Geomagnetism Program. The National Center for Atmospheric Research is sponsored by the National Science Foundation. Generous support from the International Space Science Institute (ISSI) for attending the conference in Bern was greatly appreciated. We acknowledge use of NASA/GSFC's Space Physics Data Facility's CDAWeb service, and OMNI data. The Weimer 2005 Model was developed by the Dan Weimer and the model results use here were computed using the Instant Run Feature of the Community Coordinated Modeling Center at Goddard Space Flight Center. The authors wish to thank C.A. Finn and A. Richmond for their helpful comments in the preparation of this paper.

The online version of the original article can be found under doi:10.1007/s11214-016-0271-2.

$凶$ M. Wiltberger

wiltbemj@ucar.edu

1 National Center for Atmospheric Research, 3080 Center Green Place, Boulder, CO 80301, USA 